\title{
A simple endoscopic scoring system to differentiate between duodenal adenoma and carcinoma
}

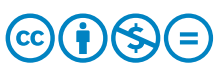

\author{
Authors \\ Institutions \\ 1 Division of Endoscopy, Shizuoka Cancer Center, \\ Shizuoka, Japan \\ 2 Division of Pathology, Shizuoka Cancer Center, \\ Shizuoka, Japan
}

Naomi Kakushima', Masao Yoshida ${ }^{1}$, Tomohiro Iwai ${ }^{1}$, Noboru Kawata ${ }^{1}$, Masaki Tanaka', Kohei Takizawa ${ }^{1}$, Sayo Ito' Kenichiro Imai ${ }^{1}$, Kinichi Hotta ${ }^{1}$, Hirotoshi Ishiwatari ${ }^{1}$, Hiroyuki Matsubayashi ${ }^{1}$, Hiroyuki Ono ${ }^{1}$, Keiko Sasaki ${ }^{2}$ to differentiate between VCL3 and VCL4 or higher. submitted 19.1.2017

accepted after revision 31.5 .2017

\author{
Bibliography \\ DOI https://doi.org/10.1055/s-0043-113567 | \\ Endoscopy International Open 2017; 05: E763-E768 \\ (c) Georg Thieme Verlag KG Stuttgart · New York \\ ISSN 2364-3722
}

Corresponding author

Naomi Kakushima MD, PhD, Division of Endoscopy,

Shizuoka Cancer Center, 1007 Shimonagakubo, Nagaizumi,

Suntougun, Shizuoka 4118777, Japan

Fax: +81-55-9895692

kakushin-tky@umin.ac.jp

\section{ABSTRACT}

Background and study aims Diagnosis of nonampullary duodenal low grade adenoma (Vienna classification category 3, VCL 3) and high grade adenoma/carcinoma (VCL 4 or higher) is important for clinical management decisions.
However, there are no criteria based on which endoscopic diagnosis can differentiate between VCL3 and VCL4 or higher. This study aimed to establish simple diagnostic criteria

Patients and methods This retrospective study included patients with superficial nonampullary duodenal epithelial tumors (NADETs) who underwent tumor resection between June 2004 and November 2016 at a single cancer center hospital. Using patient demographics and endoscopic findings from 2004 to 2013, variables related to the final histology of VCL4 or higher were analyzed, and a predictive model was developed. Validation analysis was performed on patients treated between 2014 and 2016.

Results A total of 150 lesions in 134 patients were included. Lesion diameter, reddish color, depression, heterogeneous or no nodularity, and mixed or depressed macroscopic types were significantly predictive of VCL4 or higher. A predictive score model was developed and a score of 3 points was defined as an appropriate cutoff for predicting VCL4 or higher. In the validation analysis, the accuracy rate of VCL4 or higher diagnosis was $86 \%$ when the score was $\geq 3$ points. Scores between patients with VCL3 and VCL4 or higher were significantly different $(P=0.0004)$.

Conclusions A simple and useful endoscopic scoring system was developed to preoperatively differentiate between VCL3 and VCL4 or higher among superficial NADETs.

\section{Introduction}

Recent studies have demonstrated a gradual increase in the incidence of duodenal adenocarcinoma [1,2]. Superficial nonampullary duodenal epithelial tumors (NADETs) including duodenal adenoma and carcinoma with no submucosal invasion are candidates for endoscopic resection (ER) [3-5]. Recent studies have reported the efficacy of ER for superficial NADETs [3-5]. However, due to the anatomical characteristics of the duodenum such as its narrow lumen, thin wall, and long distance from the mouth, which sometimes makes it difficult to manipulate an endoscope, duodenal ER harbors a higher risk of complication than does ER in the stomach or colon [4-7]. There- fore, the diagnosis of superficial NADETs is important when considering resection or follow-up and when choosing which method of ER to undertake in the case of resection [3].

Obtaining a biopsy from the lesion is considered to be the gold standard of diagnosis [8]. However, a biopsy may cause inadvertent submucosal fibrosis and further complicate ER [4]. In addition, diagnoses based on biopsy and resected specimens can pose considerable discrepancies $[4,5,9]$. Therefore, an endoscopy-based diagnosis is preferable for superficial NADETs that are likely to undergo ER.

Previous studies have analyzed the macroscopic characteristics of superficial NADETs and reported that a reddish color [5, 9] and depressions within the lesion $[9,10]$ are suggestive of 

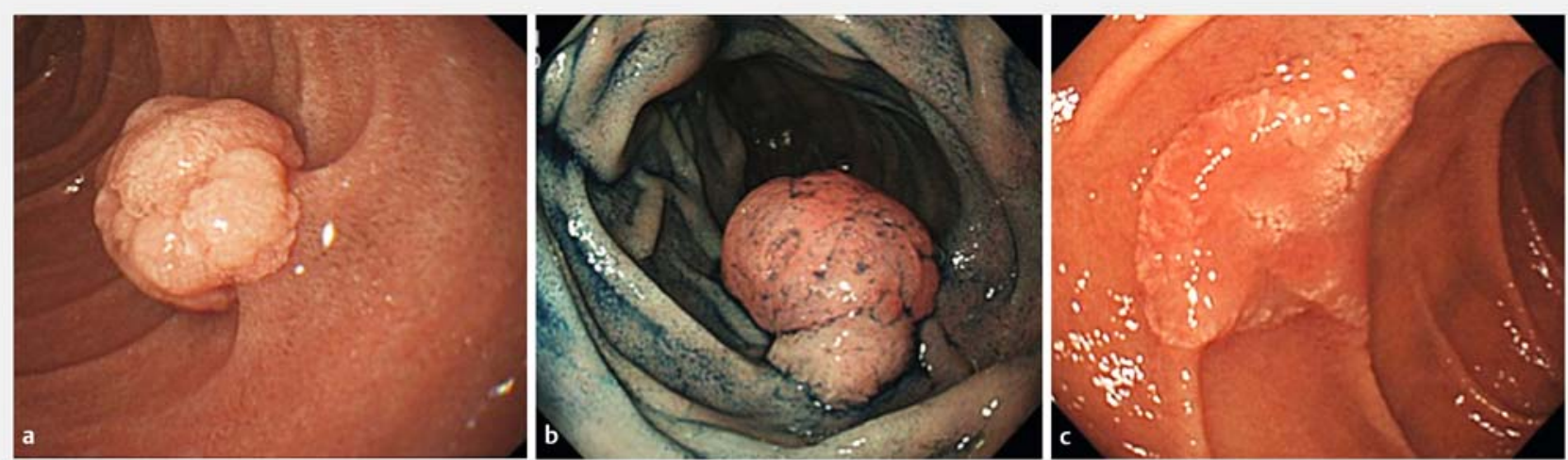

- Fig. 1 a A 10-mm white Isp lesion with uniform nodularity. b A 12-mm Is lesion with heterogenous nodularity with a reddish large component and isochromatic small component. c A 18-mm lla lesion with no nodularity. A reddish depression is observed on the surface.

carcinoma rather than adenoma. Moreover, a lesion with a biopsy-based diagnosis of high grade adenoma (Vienna classification category 4.1, VCL4.1) often yields a final diagnosis of adenocarcinoma $[5,9,10]$. However, no comprehensive diagnostic criteria have yet been proposed for the endoscopic diagnosis of duodenal carcinoma. This study aimed to clarify the endoscopic characteristics of superficial NADETs and establish simple endoscopic diagnostic criteria to differentiate between duodenal low grade adenoma (VCL3) and VCL4 or higher.

\section{Materials and methods}

\section{Patients}

This retrospective study included 150 lesions in 134 consecutive patients with superficial NADETs who underwent tumor resection by ER or surgery between June 2004 and November 2016 at Shizuoka Cancer Center Hospital. The hospital's institutional review board approved this retrospective medical record review study (25-J155-25-1-3).

\section{Derivation dataset}

Two endoscopists (N.K., M.Y.) reviewed endoscopic images of 72 lesions of superficial NADETs among 62 patients who were resected between June 2004 and December 2013. Both were board-certified endoscopists with more than 7 years of endoscopy experience. Two patients diagnosed as having familial adenomatous polyps (FAP) with 5 lesions and 1 lesion, respectively, were included. Fifty-two lesions among 72 lesions had been biopsied (median number of biopsies, 1) before examination in our hospital. All images were taken by high-resolution forward-view endoscopes (H260-GIF or H260Z-GIF, Olympus, Tokyo) without magnification. White-light images and chromoendoscopy with indigo carmine images were analyzed. If there was any inconsistency in the assessment of the endoscopic findings, a final diagnosis was decided upon by a joint review of these images. Preoperative assessment variables such as patient's age, sex, and lesion location, and endoscopic characteristics such as lesion diameter, color, presence of depression and nodularity, and macroscopic type were recorded. The macro- scopic types of superficial NADETs were classified using the Paris endoscopic classification [11]. According to endoscopic features, the macroscopic types included protruded pedunculated (Ip), protruded sessile (Is), superficial elevated (IIa), and superficial shallow or depressed types (IIC). Mixed patterns, including Ila + Is or Ila + Ilc, were diagnosed when more than one component was observed. Colors of the lesion were described as white, isochromatic or red. Nodularity of the lesion was described as "uniform" or "heterogenous" when the surface was covered with even or uneven nodules ( $\triangleright$ Fig. 1 ). If the lesion was depressed (IIC), the finding of nodularity was described as "none". Histological features were evaluated according to the revised Vienna classification of gastrointestinal epithelial neoplasia [12]. Lesions were diagnosed as VCL3, VCL4 or higher depending on the degree of cytological and architectural atypia, and invasion into the lamina propria.

Univariate analysis was performed to assess significant factors indicative of VCL4 or higher. A scoring system was established based on endoscopic characteristics to differentiate between VCL3 and VCL4 or higher. This system only included predictor variables that remained statistically significant $(P<0.05)$.

\section{Validation dataset}

The established scoring system was applied to diagnose 78 lesions of superficial NADETs among 72 patients who were treated between January 2014 and November 2016. Two patients diagnosed as having FAP with one lesion each were included. In total, 54 lesions among 78 lesions had been biopsied (median number of biopsies, 1 ) before examination in our hospital. The diagnosis based on the scoring system was compared to the final histology. Endoscopic characteristics of the lesions were recorded immediately after endoscopy performed by six endoscopists (N.K., M.Y., T.I., K. T., M.T., N.K.) who were blinded to the final histology. The score was calculated based on the recorded endoscopic characteristics. Endoscopic experience among the six endoscopists varied from 6 to 17 years. 


\section{Statistical analysis}

Descriptive statistics for all patients were generated for all measures, including medians and ranges for continuous measures, and frequencies and proportions for categorical measures. Univariate analyses were performed to examine the relationships among each of the individual patient and lesion measures and VCL3 or VCL4 or higher. Chi-squared and Fisher's exact tests were used to calculate statistical significance for categorical predictors, and the Mann-Whitney $U$ test was used for continuous predictors. All analyses were performed using Excel statistics 2012 (Social Survey Research Information, Tokyo, Japan).

\section{Results}

\section{Factors indicative of Vienna classification category 4 or higher}

Preoperative assessment variables of 72 lesions according to the final histology of VCL3 or VCL4 or higher are shown in $\mathbf{T a}$ ble 1. Of these 72 lesions, 10 were VCL3, 5 were VCL4.1, 51 were intramucosal carcinoma (VCL4.4), and 6 were submucosal invasive carcinoma (VCL5). The median diameters of lesions preoperatively assessed by endoscopy were 10 and $15 \mathrm{~mm}$ for VCL3 and VCL4 or higher, respectively. Lesion diameter, color, macroscopic type, presence of depression, and heterogeneous or no nodularity were predictive factors for VCL4 or higher $(P<$ 0.05).

\section{Scoring system to predict VCL4 or higher}

A predictive scoring system was created by allocating points from 0 to 2 to each endoscopic finding, as shown in $>$ Table 2 . When a lesion had mixed color, the color with the highest point was selected. A box plot of scores allocated for each endoscopic finding and final diagnoses as VCL3 or VCL4 or higher is shown in $>$ Fig. 2. The median scores for VCL3 and VCL4 or higher were 1 and 4, respectively. Lesions diagnosed as VCL4 or higher had significantly higher scores than those diagnosed as VCL3 (Mann-Whitney $U$ test, $P=0.0001$ ). To investigate the relationship between the scoring system and the final diagnosis of VCL3 or VCL4 or higher, a receiver operating characteristic (ROC) curve was derived. On the basis of the ROC curve, a score of 3 points was defined as an appropriate cutoff for predicting VCL4 or higher with a sensitivity rate of 0.84 , specificity rate of 0.90 , and a false positive rate of 0.1 ( $\triangleright$ Fig.3).

\section{Validation analysis}

To test the validity of our predictive model, we examined 78 additional lesions in 72 patients treated between January 2014 and November 2016. The data for these patients were not used in the process of building our scoring system. Of these 78 lesions, 14 were VCL3, 4 were VCL4.1, 56 were VCL4.4, and 4 were VCL5. When the lesion had a total score of 3 points or more, the sensitivity, specificity, and accuracy of diagnosing VCL4 or higher were $88 \%, 79 \%$, and $86 \%$, respectively. A box plot of scores and final diagnoses as VCL3 or VCL4 or higher is shown in $>$ Fig. 4 . The difference in scores among the groups
- Table 1 Preoperative assessment variables of 72 superficial nonampullary duodenal epithelial tumors.

\begin{tabular}{|c|c|c|c|}
\hline Variable & VCL3 & $\begin{array}{l}\text { VCL4 or } \\
\text { higher }\end{array}$ & $P$ value \\
\hline \multicolumn{4}{|l|}{ Sex } \\
\hline - Male & 9 & 49 & 0.7 \\
\hline - Female & 1 & 13 & \\
\hline Age, median (range) & $67(34-81)$ & $61(27-84)$ & 0.21 \\
\hline \multicolumn{4}{|c|}{ Location (portion of the duodenum) } \\
\hline - 1st & 3 & 7 & 0.11 \\
\hline . 2nd & 7 & 49 & \\
\hline - $3 r d$ & 0 & 6 & \\
\hline $\begin{array}{l}\text { Lesion diameter, } \\
\text { median (range), mm }\end{array}$ & $10(6-25)$ & $15(4-50)$ & 0.013 \\
\hline \multicolumn{4}{|l|}{ Color } \\
\hline - White & 7 & 11 & 0.0003 \\
\hline - Isochromatic & 3 & 10 & \\
\hline - Red & 0 & 41 & \\
\hline \multicolumn{4}{|l|}{ Macroscopic type } \\
\hline - Is, Ip, Ila & 9 & 27 & 0.006 \\
\hline - Mixed or llc & 1 & 35 & \\
\hline \multicolumn{4}{|l|}{ Depression } \\
\hline - No & 9 & 30 & 0.014 \\
\hline - Yes & 1 & 32 & \\
\hline \multicolumn{4}{|l|}{ Nodularity } \\
\hline - Uniform & 7 & 12 & 0.0007 \\
\hline $\begin{array}{l}\text { - Heterogeneous or } \\
\text { none }\end{array}$ & 3 & 50 & \\
\hline
\end{tabular}

VCL3, Vienna classification category 3; VCL4, Vienna classification category 4.

- Table 2 Scoring system for VCL3 and VCL4 or higher.

\begin{tabular}{|l|l|l|l|}
\hline $\begin{array}{l}\text { Endoscopic } \\
\text { finding }\end{array}$ & Score & \\
\hline & $\mathbf{0}$ & $\mathbf{1}$ & $\mathbf{2}$ \\
\hline Lesion diameter & $<10 \mathrm{~mm}$ & $\geq 10 \mathrm{~mm}$ & \\
\hline Color & White & Isochromatic \\
\hline Macroscopic & $\begin{array}{l}\text { Is, Ip, lla with- } \\
\text { out depression }\end{array}$ & $\begin{array}{l}\text { Any type with depres- } \\
\text { sion or mixed type }\end{array}$ \\
\hline Nype & Uniform & $\begin{array}{l}\text { Heterogeneous or } \\
\text { none }\end{array}$ \\
\hline
\end{tabular}




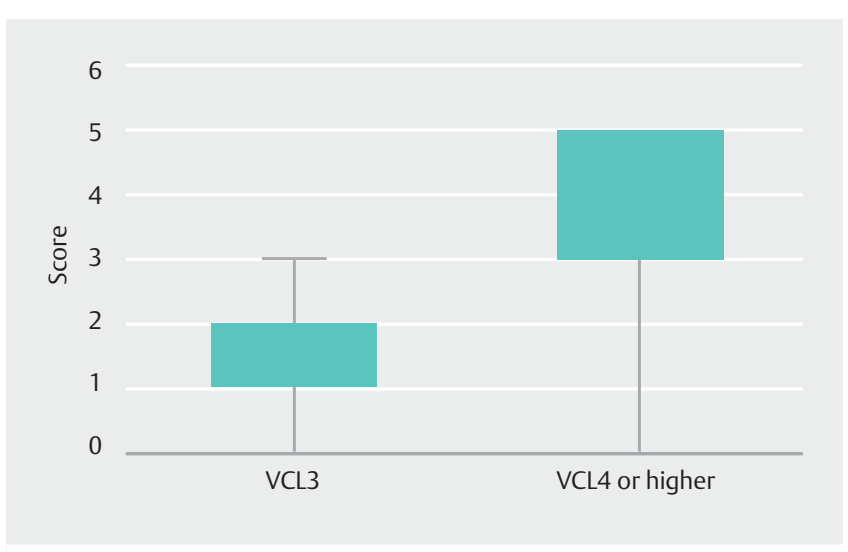

- Fig. 2 A box plot of the scores of lesions with a final histology of Vienna classification category 3 (VCL3) or VCL4 or higher. The difference in the scores between the two groups was significant (Mann-Whitney $U$ test, $P=0.0001$ ).

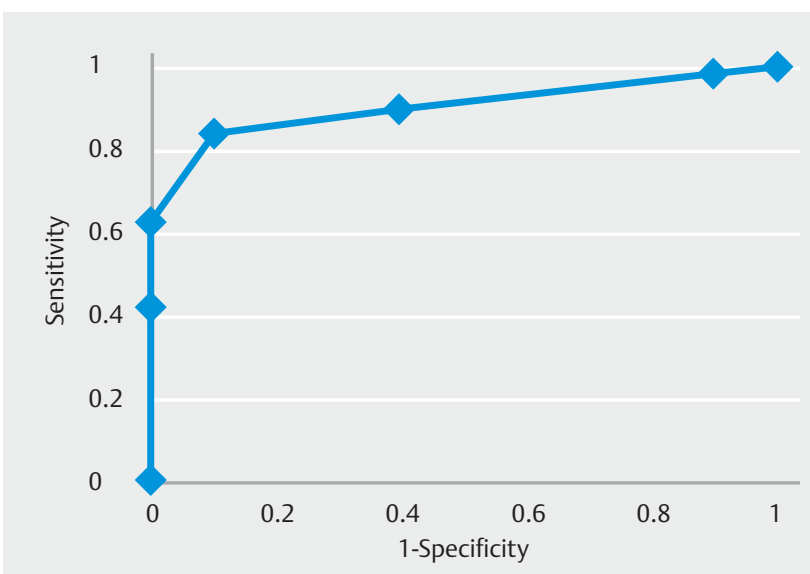

- Fig. 3 A receiver operating characteristic curve was derived and a score of 3 points was defined as an appropriate cutoff for predicting Vienna classification category 4 or higher.

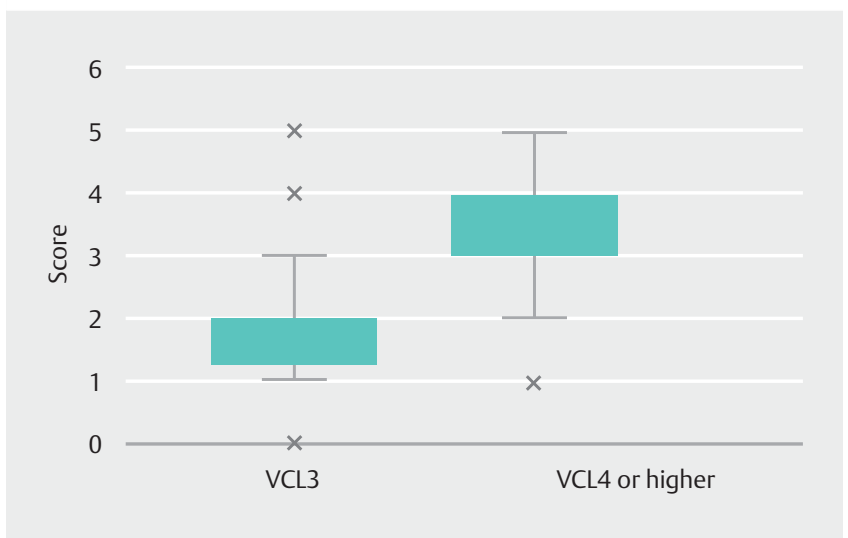

- Fig. 4 A box plot of the scoring system in the validation analysis. The difference in the scores between the two groups was significant (Mann-Whitney $U$ test, $P=0.0001$ ). was statistically significant at $P=0.0001$ (Mann-Whitney $U$ test).

\section{Lesions inconsistent with the scoring system}

In the validation analysis, three lesions in three patients who had a score of 3 points or more had a final histology of VCL3. The first lesion was a $6-\mathrm{mm}$ red Ila lesion with no nodularity. The scoring system allocated 3 points, but the subjective impression of the endoscopist was that the lesion was adenoma. This lesion was followed previously for 2 years before resection and the color changed from white to red. The second lesion was a 5-mm red Ilc lesion with no nodularity. The scoring system allocated 4 points and the subjective impression of the endoscopist was carcinoma. The final lesion was a $12-\mathrm{mm}$ partially red Ila + Ilc lesion with no nodularity. The scoring system allocated 5 points and the subjective impression of the endoscopist was carcinoma. All three patients underwent ER.

Eight lesions in eight patients who had a score of $<3$ points had a final histology of VCL4 or higher. The color was isochromatic in 2 lesions and white in 6 lesions. The macroscopic types were Ilc for 1 lesion, Is for 1 lesion, and the remainder were Ila lesions. Nodularity was heterogeneous or not found in 6 lesions. The subjective impression of the endoscopist was adenoma in 5 lesions. The other 3 lesions were diagnosed as carcinoma regardless of the score because of the heterogeneous appearance of the lesion. According to the final pathology, 1 lesion had a focal high grade component in a VCL3, 6 lesions were VCL4.4, and 1 lesion showed carcinoma with minute submucosal invasion (VCL5). The submucosal carcinoma was a 7-mm white Ila lesion with a score of 1 point. This patient underwent partial duodenectomy because he had 2 lesions in the 2nd and 3 rd portions of the duodenum, which were considered difficult for ER. Of the remaining 7 patients, 6 underwent ER, and 1 underwent a partial duodenectomy.

\section{Discussion}

From previous studies, it is considered that most primary nonampullary duodenal carcinomas (NADCs) follow the adenomacarcinoma sequence [13-15]. Focal carcinoma has been recognized in adenomatous polyps [13], and residual adenomatous tissue has been recognized in surgically resected duodenal carcinoma [13-15]. A follow-up study including patients with superficial NADETs demonstrated that the majority of VCL3 showed no progression during follow-up, but $21 \%$ showed progression to VCL4 or higher [10]. Therefore, diagnosing VCL3 from VCL4 or higher among NADETs is essential to decide clinical management.

In this study, we created a simple scoring system using endoscopic findings to distinguish between VCL3 and VCL4 or higher. The endoscopic findings could be assessed using only white-light imaging (WLI) with or without chromoendoscopy. A score of 3 points or more correlated strongly with the final pathology of VCL4 or higher. The endoscopic findings of reddish color, the presence of depression, loss of nodularity, and mixed macroscopic types are also important in diagnosing carcinoma in the stomach or colorectum [16-20]. Therefore, it is 
acceptable for endoscopists who are familiar with diagnosing carcinoma of the stomach or the colorectum to retrieve these WLI findings among superficial NADETs.

The accuracy of diagnosing VCL4 or higher using our scoring system was $86 \%$ in the validation study. A similar result has been reported in a retrospective multicenter study with $75 \%$ accuracy when using endoscopic features such as reddish color, nodular/rough surface, and depressed portion [5]. Considering that the reported accuracy of biopsy-based diagnosis is $68 \%-$ $74 \%[5,9]$, it is important to make an endoscopic diagnosis before taking a biopsy. The merit of endoscopic diagnosis is that we can judge the lesion from the whole image, whereas biopsies have issues of false negatives when they are performed in an area with a lower grade of histology, or the retrieved specimen may not be sufficient for evaluation. Total biopsy for small lesions may be quick, but it is sometimes difficult for large lesions and for patients with multiple lesions. As heterogeneity of histologic grade within a single lesion is not rare $[3-5,9$, 18], endoscopic diagnosis may facilitate biopsies from areas with higher grades of histology.

The use of narrow-band imaging (NBI) with or without magnified endoscopy for the diagnosis of superficial NADETs has been reported in a few small studies [21-23]. Yoshimura et al. reported that $\mathrm{NBI}$ with magnified endoscopy following WLI might be a useful method to predict histological grade of NADETs [21]. Kikuchi et al. assessed surface and vascular patterns of NADETs and proposed a diagnostic algorithm to differentiate histological grade of NADETs [22]. On the other hand, Tsuji et al. reported that there was no significant difference in microvascular patterns between VCL3 and VCL4 lesions, but an irregular surface pattern was more often observed among VCL4 lesions [23]. Although the diagnosis using NBI for NADETs seems promising, it is still not standardized and its superiority compared to WLI diagnosis still requires further evaluation.

In the validation study, one lesion with submucosal invasion had a score of 1 point. Duodenal carcinoma with invasion limited to the submucosa is extremely rare, and it has been reported that reddish color is a common factor, but the prediction of submucosal invasion is difficult [5]. In our case, the invasion depth was minute, the lesion was white without depression, and we could not find any endoscopic features suggestive of submucosal invasion even after re-evaluation of the endoscopic image.

Our study included four patients with FAP having a total of nine lesions. We used the same diagnostic system for both sporadic and FAP associated NADETs. Patients with FAP are known to have a high prevalence of NADETs [24,25]. Both sporadic and FAP associated duodenal carcinoma may occur because of the adenoma carcinoma sequence, and thus macroscopic types of NADET are assumed to be similar. However, this should be confirmed in further studies.

The first limitation of our study is that the number of VCL3 lesions included was relatively small. As we only included lesions that were resected, some lesions with an endoscopic or biopsy diagnosis of VCL3 may have been followed without resection. Thus, the possibility of selection bias cannot be ignored. Another limitation is that endoscopic findings of color or macroscopic type may be influenced by biopsy. There is a possibility that the color may change to red, and a depression may appear after taking a biopsy. However, biopsy-induced changes are often focal or limited and sometimes are accompanied by regenerative mucosa or slight fold convergence. Therefore, we should be careful to observe the whole lesion to extract appropriate endoscopic findings with consideration for artificial changes caused by previous biopsies. Finally, this is a retrospective study performed in a single institution. We showed good reproducibility of the scoring system in our own validation study; however, this should be confirmed in a prospective, multicenter study.

In conclusion, a simple and useful endoscopic scoring system was developed to differentiate preoperatively between VCL3 and VCL4 or higher among superficial NADETs.

\section{Competing interests}

\section{None}

\section{References}

[1] Bilimoria KY, Bentrem DJ, Wayne JD et al. Small bowel cancer in the United States. Ann Surg 2009; 249: 63-71

[2] Gustafsson B, Siddique L, Chan A et al. Uncommon cancers of the small intestine, appendix and colon: an analysis of SEER 1973-2004, and current diagnosis and therapy. Int J Oncol 2008; 33: 1121-1131

[3] Kakushima N, Ono H, Takao T et al. Method and timing of resection of superficial non-ampullary duodenal epithelial tumors. Dig Endosc 2014; $26: 3-40$

[4] Kakushima N, Kanemoto H, Tanaka M et al. Treatment for superficial nonampullary duodenal epithelial tumors. World J Gastroenterol 2014; 20: $12501-12508$

[5] Goda K, Kikuchi D, Yamamoto Y et al. Endoscopic diagnosis of superficial nonampullary duodenal epithelial tumors in Japan: Multicenter case series. Dig Endosc 2014; 26: 23-29

[6] Inoue T, Uedo N, Yamashina T et al. Delayed perforation: a hazardous complication of endoscopic resection for non-ampullary duodenal neoplasm. Dig Endosc 2014; 26: 220-227

[7] Kakushima N. Hurdles of duodenal endoscopic submucosal dissection, delayed bleeding and perforation. Dig Endosc 2015; 27 : 298 299

[8] Basford PJ, Bhandari P. Endoscopic management of nonampullary duodenal polyps. Therap Adv Gastroenterol 2012; 5: 127 -138

[9] Kakushima N, Kanemoto H, Sasaki K et al. Endoscopic and biopsy diagnoses of superficial nonampullary duodenal adenocarcinomas. World J Gastroenterol 2015; 21: 5560 - 5567

[10] Okada J, Fujisaki J, Kasuga A et al. Sporadic nonampullary duodenal adenoma in the natural history of duodenal cancer: a study of followup surveillance. Am J Gastroenterol 2011; 106: 357-364

[11] The Paris endoscopic classification of superficial neoplastic lesions: esophagus, stomach, and colon: November 30 to December 1, 2002. Gastrointest Endosc 2013; 58: S3-43

[12] Dixon MF. Gastrointestinal epithelial neoplasia: Vienna revisited. Gut 2002; 51: $130-131$

[13] Perzin KH, Bridge MF. Adenomas of the small intestine: A clinicopathologic review of 51 cases and a study of their relationship to carcinoma. Cancer 1981; 48: 799-819 
[14] Sellner F. Investigations on the significance of the adenoma-carcinoma sequence in the small bowel. Cancer 1990; 66: $702-715$

[15] Seifert E, Schulte F, Stolte M. Adenoma and carcinoma of the duodenum and papilla of Vater: A clinicopathologic study. Am J Gastroenterol 1992; 87: 37-42

[16] Kitoh T, Yanai $H$, Matsubara $Y$ et al. Endoscopic findings potentially predictive of gastric cancer in borderline lesions diagnosed by forceps biopsy. Hepatogastroenterology 2005; 52: 404-408

[17] Tsuchigame T, Ogata Y, Sumi M et al. Differential diagnosis of gastric adenoma and type lla early gastric cancer. Acta Radiol 1991; 32: 37 41

[18] Takao M, Kakushima N, Takizawa N et al. Discrepancies in histologic diagnoses of early gastric cancer between biopsy and endoscopic mucosal resection specimens. Gastric Cancer 2012; 15: 91 - 96

[19] Kudo S. Endoscopic mucosal resection of flat and depressed types of early colorectal cancer. Endoscopy 1993; 25: 455-461

[20] Ikehara H, Saito Y, Matsuda T et al. Diagnosis of depth of invasion for early colorectal cancer using magnifying colonoscopy. J Gastroenterol Hepatol 2010; 25: 905-912
[21] Yoshimura N, Goda K, Tajiri H et al. Endoscopic features of nonampullary duodenal tumors with narrow-band imaging. Hepatogastroenterology 2010; 57: $462-467$

[22] Kikuchi D, Hoteya S, lizuka T et al. Diagnostic algorithm of magnifying endoscopy with narrow band imaging for superficial non-ampullary duodenal epithelial tumors. Dig Endosc 2014; 26: 16-22

[23] Tsuji S, Doyama H, Tsuji K et al. Preoperative endoscopic diagnosis of superficial non-ampullary duodenal epithelial tumors, including magnifying endoscopy. World J Gastroenterol 2015; 21: 11832 11841

[24] Vasen HF, Möslein G, Alonso A et al. Guidelines for the clinical management of familial adenomatous polyposis (FAP). Gut 2008; 57 : $704-713$

[25] Spigelman AD, Talbot IC, Penna C et al. Evidence for adenoma-carcinoma sequence in the duodenum of patients with familial adenomatous polyposis. The Leeds Castle Polyposis Group (Upper Gastrointestinal Committee). J Clin Pathol 1994; 47: 709-710 